\title{
Effets de la vasopressine sur le profil moteur du réticulo-rumen chez le mouton
}

\author{
$H$ Brugère, $H$ Combrisson \\ Laboratoire de Physiologie-Thérapeutique, Ecole Nationale Vétérinaire, \\ 94700 Maisons-Alfort, France
}

Summary - Intravenous injections of lysine-vasopressin into sheep inhibited rumino-reticular phasic contractions and electric activity. Sometimes, a rapid electric pattern was observed and persisted even after reappearance of normal phasic contractions.

Introduction - Chez les ruminants adultes, les liquides administrés par voie buccale tombent généralement dans le rumen. II est possible de favoriser leur passage vers la caillette en soumettant les animaux à un jeûne hydrique prolongé, ou par administration orale de sulfate de cuivre. Ce résultat peut aussi être obtenu en injectant par voie veineuse une forte dose de vasopressine qui produit, pendant environ $10 \mathrm{~min}$, un état de fermeture de la gouttière œsophagienne tel que les solutions de marqueurs (glucose, par exemple, Brugère et al, 1987; Mikhail et al, 1988) ou des médicaments destinés à l'intestin (Mikhail, 1986) pourront court-circuiter le rumen. Ayant constaté (Brugère et al, 1987) que les effets de la vasopressine s'accompagnent aussi de l'inhibition de la motricité du réticulo-rumen, ce travail a été entrepris pour en préciser les caractéristiques.

Matériel et Méthodes - Cinq moutons de race $\|$ e-de-France ont été préparés en vue d'une étude électromyographique : l'extrémité dénudée de fils d'acier $\mathrm{Ni}-\mathrm{Cr}$ isolés est insérée dans la musculeuse du réseau et du rumen selon la technique de Ruckebusch (1970).
Parmi eux, 2 animaux ont subi une transposition du réseau en région sous-cutanée (Bost, 1958), et chez un autre, la force des contractions était enregistrée par l'intermédiaire de jauges de contrainte laissées à demeure. La lysine-vasopressine était injectée par l'intermédiaire d'un cathéter jugulaire aux doses de 0,01 a $0,5 \mathrm{UI} / \mathrm{kg}$. Les enregistrements étaient pratiqués sur les animaux $1 \mathrm{~h}$ après le repas.

Résultats et Discussion - La vasopressine produit une inhibition marquée des activités électriques et mécaniques phasiques du réseau. Après une période d'arrêt de $4,5 \mathrm{~min}$ pour $0,1 \mathrm{Ul} / \mathrm{kg}$ et de $9,5 \mathrm{~min}$ pour $0,5 \mathrm{UI} / \mathrm{kg}$, la motricité retrouve graduellement sa valeur basale. La durée de la phase d'arrêt, dépendante de la dose, est réduite par la répétition des injections. L'évolution de la fréquence est variable: elle peut dépasser le rythme initial, ou présenter une seconde phase de ralentissement. L'effet de ralentissement s'accompagne d'une réduction de l'amplitude des contractions. L'activité du rumen est réduite à des contractions secondaires apparaissant pendant la phase d'arrêt du réseau. Les contractions primaires reprennent parallèlement à celles du réseau. 


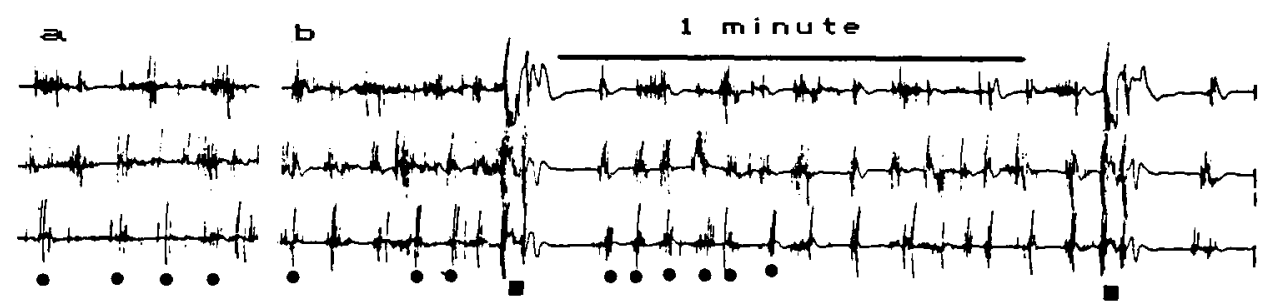

Fig 1. Activité électrique du réseau après injection de 0,25 Ui/kg de vasopressine. a : au cours de la période d'inhibition motrice, salves de potentiels attribuables à l'activité intrinsèque (O). b : persistance de ces salves au cours de la reprise des contractions biphasiques normales caractérisées par deux salves de potentiels de grande amplitude (B).

A plusieurs reprises on a observé l'activité suivante : pendant la phase d'arrêt des contractions phasiques du réticulum, le tracé électrique présente des salves de potentiels, synchrones pour les sites d'enregistrement, à la fréquence de 10 à 15 salves/min (fig 1). Cette activité est analogue à celle décrite pour le rumen après vagotomie par Ruckebusch (1986). L'observation visuelle chez les animaux porteurs d'une poche sous-cutanée montre l'existence de fasciculations de très faible amplitude. Lorsque des contractions phasiques reprennent, les potentiels correspondants s'intercalent entre les salves de faible amplitude (fig 1). La présence de cette activité est de 20,30 et 34 min pour des doses de $0,1,0,25$ et $0,5 \mathrm{Ul} / \mathrm{kg}$.

Ces modifications motrices sont observées à des doses de lysine-vasopressine équivalentes à celles qui produisent la fermeture de la gouttière cesophagienne. L'inhibition de la motricité phasique du réseau est, comme la relaxation de l'orifice réticulo-omasal, une condition favorable au transit de liquide dans la gouttière : elle est également observée lors de la déglutition spontanée de liquides et après ingestion de sulfate de cuivre (Tsiamitas et Brikas, 1981). Les effets de la vasopressine présentent d'ailleurs une certaine analogie avec ceux décrits après administration de sulfate de cuivre, en l'occurrence une activité électrique représentée par des salves de potentiels sur le réseau, de fréquence élevée et non associée à une activité phasique. Ce type d'activité électrique, exagéré dans la distension, correspondrait à une variation du tonus de l'organe (Ruckebusch, 1986).

Bost J (1958) J Physiol (Paris) 50, 170-171

Brugère $\mathrm{H}$ et al (1987) Rec Méd Vét 163, 857864

Mikhail M (1986) Inaugural Dissertation, Tierarztliche Hochschule, Hannovre

Mikhail M et al (1988) Am J Vet Res 49, 17131715

Ruckebusch Y (1970) J Physio/ 210, 857-882

Ruckebusch Y (1986) Ann Méd Vét 130, 613625

Tsiamitas C, Brikas P (1981) Ann Rech Vet 12, 117-121 